\title{
Dynamical mass generation in Landau gauge QCD *
}

\author{
C. S. Fischer ${ }^{\mathrm{a}}$, F. Llanes-Estrada ${ }^{\mathrm{b}}$ and R. Alkofer ${ }^{\mathrm{c}}$ \\ ${ }^{a}$ IPPP, University of Durham, Durham DH1 3LE, U.K. \\ ${ }^{\mathrm{b}}$ Fisica Teorica I, Univ. Complutense, Madrid 28040, Spain \\ cInstitute for Theoretical Physics, University of Tübingen, D-72076 Tübingen, Germany
}

We summarise results on the infrared behaviour of Landau gauge QCD from the Green's functions approach and lattice calculations. Approximate, nonperturbative solutions for the ghost, gluon and quark propagators as well as first results for the quark-gluon vertex from a coupled set of Dyson-Schwinger equations are compared to quenched and unquenched lattice results. Almost quantitative agreement is found for all three propagators. Similar effects of unquenching are found in both approaches. The dynamically generated quark masses are close to 'phenomenological' values. First results for the quark-gluon vertex indicate a complex tensor structure of the non-perturbative quark-gluon interaction.

\section{INTRODUCTION}

The nonperturbative properties of the quarkgluon interaction are at the heart of the most interesting phenomena of QCD such as confinement and dynamical chiral symmetry breaking. On a fundamental level the details of the quarkgluon interaction are encoded in the one-particle irreducible quark-gluon vertex. Together with the quark and gluon propagators this vertex enters as a vital ingredient in model building and plays a key role in bridging the gap between the coloured fundamental degrees of freedom of the theory and the observed colour-neutral hadron states. On a phenomenological level the quarkgluon interaction manifests itself in the details of the quark-(anti-)quark potential. Relativistic effects play an important role in potential models and the proper choice of the Lorentz structure of the quark-antiquark interaction is vital to guarantee the agreement of the theoretical predictions with the experimental data. Ultimately, a derivation of these structures from the underlying quark-gluon interaction is mandatory. To this end one has to know the properties of the most fundamental Green's functions of QCD, the dressed propagator and vertex functions.

\footnotetext{
* Summary of a talk given at the international conference QCD DOWN UNDER, March 10 - 19, Adelaide, Australia
}

The infrared behaviour of the propagators of Landau gauge QCD has been investigated extensively over the past years in both, lattice Monte Carlo simulations and the continuum Green's functions approach. Lattice simulations are the only $a b$ initio method known so far and are by now precise enough to pin down these propagators accurately in a large momentum range centered around $1 \mathrm{GeV}$. In the deep infrared, however, lattice results are inevitably plagued by finite volume effects. In the continuum formulation of QCD the Dyson-Schwinger equations (DSEs) provide a tool complementary to lattice simulations. They can be solved analytically in the infrared. Furthermore numerical solutions over the whole momentum range are available by now. The truncation assumptions necessary to close the DSEs can be checked in the momentum regions where lattice results are available. In general results from DSEs have the potential to provide a successful description of hadrons in terms of quarks and gluons, see [1-3] and references therein. 


\section{LANDAU GAUGE QCD}

The ghost, gluon and quark propagators, $D_{G}(p), D_{\mu \nu}(p)$ and $S(p)$, in Euclidean momentum space can be generically written as

$$
\begin{aligned}
D_{G}\left(p, \mu^{2}\right) & =-\frac{G\left(p^{2}, \mu^{2}\right)}{p^{2}}, \\
D_{\mu \nu}\left(p, \mu^{2}\right) & =\left(\delta_{\mu \nu}-\frac{p_{\mu} p_{\nu}}{p^{2}}\right) \frac{Z\left(p^{2}, \mu^{2}\right)}{p^{2}}, \\
S\left(p, \mu^{2}\right) & =\frac{1}{-i \not p A\left(p^{2}, \mu^{2}\right)+B\left(p^{2}, \mu^{2}\right)} \\
& =\frac{Z_{Q}\left(p^{2}, \mu^{2}\right)}{-i \not p+M\left(p^{2}\right)} .
\end{aligned}
$$

Here $\mu^{2}$ denotes the renormalisation scale and $G\left(p^{2}, \mu^{2}\right)$ and $Z\left(p^{2}, \mu^{2}\right)$ are the ghost and gluon dressing functions. The Dyson-Schwinger equations for these dressing functions have been solved in their continuum formulation [4,5] as well as on a torus, i.e. employing periodic boundary conditions [6]. In the continuum they can be solved analytically in the infrared and one finds simple power laws,

$$
\begin{aligned}
& Z\left(p^{2}, \mu^{2}\right) \sim\left(p^{2} / \mu^{2}\right)^{2 \kappa}, \\
& G\left(p^{2}, \mu^{2}\right) \sim\left(p^{2} / \mu^{2}\right)^{-\kappa},
\end{aligned}
$$

for the gluon and ghost dressing function with exponents related to each other. Hereby $\kappa$ is an irrational number, $\kappa=(93-\sqrt{2101}) / 98 \approx 0.595$ which depends slightly on the truncation scheme $[7,8]$. Note that $\kappa>0$, also shown in [9], implies the dominance of the 'geometric' ghost degrees of freedom in the infrared in agreement with the Kugo-Ojima confinement criterion and Zwanziger's horizon condition [10-12]. Recently these results from the Dyson-Schwinger approach have been confirmed independently in studies of the exact renormalisation group equation [13].

A combination of the ghost and gluon dressing functions can be used to define the nonperturbative running coupling [4]

$\alpha\left(p^{2}\right)=\alpha\left(\mu^{2}\right) G^{2}\left(p^{2}, \mu^{2}\right) Z\left(p^{2}, \mu^{2}\right)$.

No vertex function appears in this definition; a fact that can be traced back to the ultraviolet finiteness of the ghost-gluon vertex in Landau

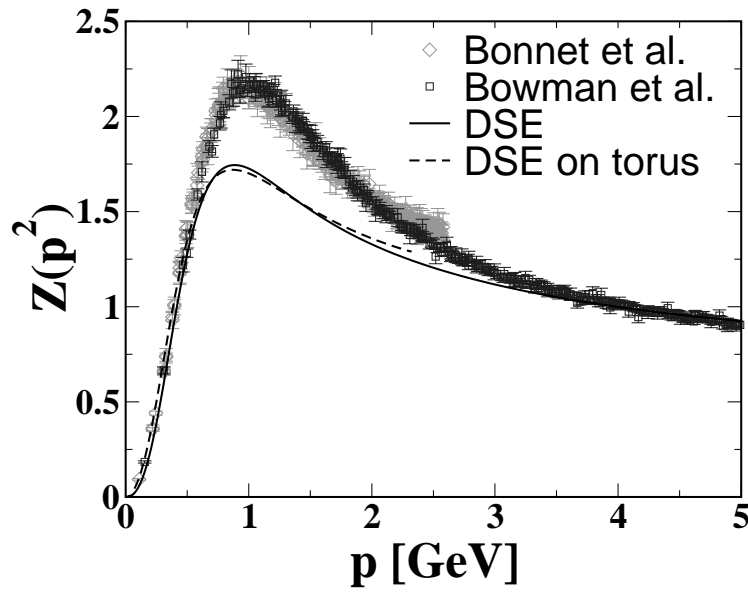

Figure 1. The gluon dressing function $Z\left(p^{2}\right)$ from DSEs $[5,6]$ and on the lattice $[17,18]$.

gauge. Note that the right hand side of this equation is a renormalisation group invariant, i.e. $\alpha\left(p^{2}\right)$ does not depend on the renormalisation point. A further renormalisation group invariant is the quark mass function, given by $M\left(p^{2}\right)=B\left(p^{2}, \mu^{2}\right) / A\left(p^{2}, \mu^{2}\right)$. From the power laws Eq. (4) one finds that the coupling has a fixed point in the infrared given by

$$
\alpha(0)=\frac{4 \pi}{6 N_{c}} \frac{\Gamma(3-2 \kappa) \Gamma(3+\kappa) \Gamma(1+\kappa)}{\Gamma^{2}(2-\kappa) \Gamma(2 \kappa)} \approx 2.972
$$

for the gauge group $\mathrm{SU}(3)$. The infrared dominance of the ghosts can be used to show that $\alpha(0)$ depends only weakly on the dressing of the ghost-gluon vertex and not at all on other vertex functions [7].

The running coupling as it results from numerical solutions for the gluon, ghost and quark propagators can be quite accurately fitted by the relatively simple function [14]

$$
\begin{aligned}
\alpha_{\mathrm{fit}}\left(p^{2}\right)= & \frac{\alpha(0)}{1+p^{2} / \Lambda_{\mathrm{QCD}}^{2}}+\frac{4 \pi}{\beta_{0}} \frac{p^{2} / \Lambda_{\mathrm{QCD}}^{2}}{1+p^{2} / \Lambda_{\mathrm{QCD}}^{2}} \\
& \times\left(\frac{1}{\ln \left(p^{2} / \Lambda_{\mathrm{QCD}}^{2}\right)}-\frac{1}{p^{2} / \Lambda_{\mathrm{QCD}}^{2}-1}\right)(6)
\end{aligned}
$$

with $\beta_{0}=\left(11 N_{c}-2 N_{f}\right) / 3$. Note that, following Ref. [15], the Landau pole has been subtracted. The scale $\Lambda_{\mathrm{QCD}}$ is hereby determined by 


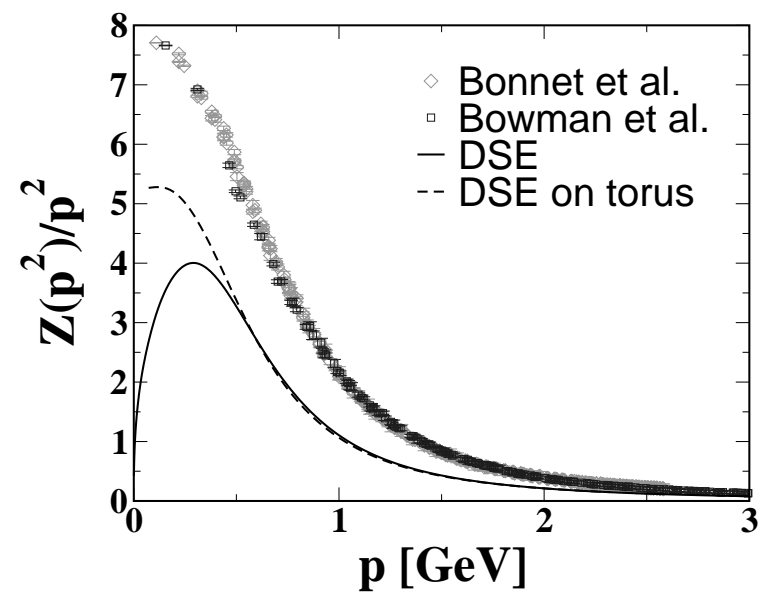

Figure 2. The gluon propagator $Z\left(p^{2}\right) / p^{2}$ from DSEs $[5,6]$ and on the lattice $[17,18]$.

fixing the running coupling at a certain scale, e.g. $\alpha_{S}\left(M_{Z}^{2}\right)=0.118$.

The numerical solutions for the gluon dressing function $Z\left(p^{2}\right)$ in the continuum and on the torus are compared to the results of recent lattice simulations $[17,18]$ in Fig. 1. The qualitative agreement with the lattice results is very good and the quantitative discrepancies in the momentum region around $1 \mathrm{GeV}$ well understood: gluon self interactions not contained in the DSE-truncation play an important role here. In the infrared both approaches agree nicely. An interesting qualitative difference, however, can be seen in Fig. 2, where we show the propagator $Z\left(p^{2}\right) / p^{2}$ instead of the dressing function. Both, the lattice results and the DSE-solution on the compact manifold tend towards a constant in the infrared, whereas the propagator from the DSEs in continuum formulation vanishes. Both DSE-solutions employ the same truncation scheme, thus the differences between them have to be attributed to the periodic boundary conditions and the finite volume on the torus. These properties of the compact space might also account for the constant gluon propagator found on the lattice.

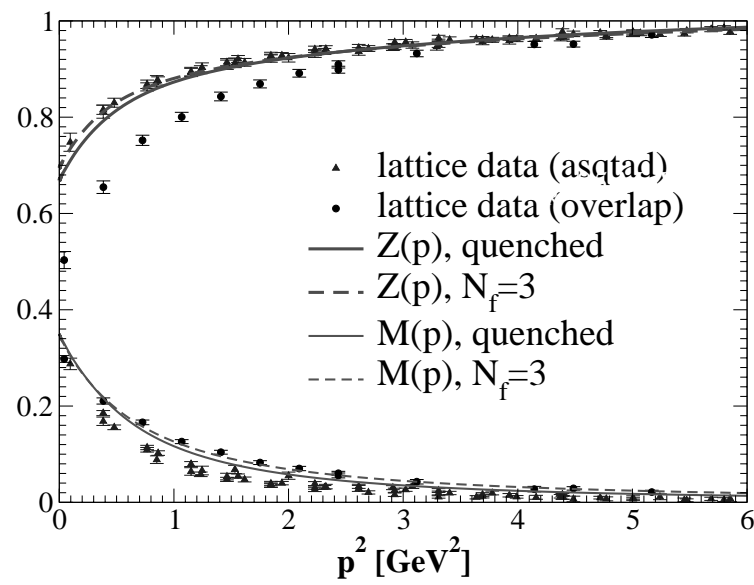

Figure 3. The quenched and unquenched quark mass function $M\left(p^{2}\right)$ and the wave function $Z_{Q}\left(p^{2}\right)$ from the DSE approach [14] compared to results from quenched lattice calculations [16].

\section{DYNAMICAL MASS GENERATION}

The dynamical generation of quark masses can be studied in detail using Dyson-Schwinger equation for the quark propagator, Eq. (3). It is a genuinely non-perturbative phenomenon and requires a careful treatment of the quark-gluon interaction employed. In the DSE-framework of Ref. [14] an ansatz for the quark-gluon vertex has been chosen that satisfies important constraints: it is genuinely nonperturbative in nature, guarantees multiplicative renormalisability in the quark DSE and has the correct limit in the perturbative momentum domain. Its structure is such that it factorizes in an Abelian and a non-Abelian part,

$\Gamma_{\nu}(q, k)=V_{\nu}^{A b e l}(p, q, k) W^{\neg A b e l}(p, q, k)$,

with $p$ and $q$ denoting the quark momenta and $k$ the gluon momentum. For the Abelian part $V_{\nu}^{\text {Abel }}$ carrying all the tensor structure the CurtisPennington construction [19] has been chosen which has been used frequently in QED. This choice has the advantage that vector as well as scalar tensor components are taken into account and their effect on the quark propagator can be studied. In fact, as detailed in Refs. [20], the inclusion of the scalar parts of the vertex is ca- 


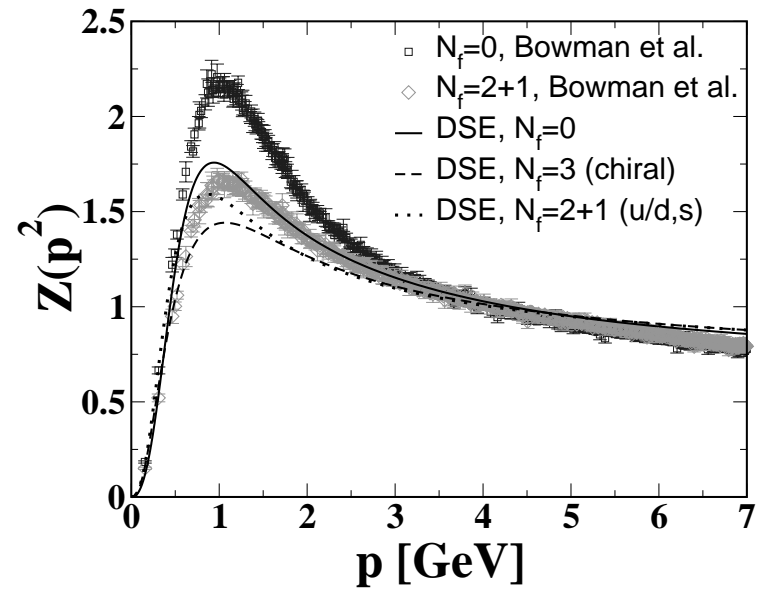

Figure 4. The quenched and unquenched gluon dressing function from the DSE approach [14] compared to results from unquenched lattice calculations [18].

pable of changing the analytic structure of the quark propagator dramatically, leading to a positive definite spectral function for the quarks.

The non-Abelian part $W^{\neg A b e l}(p, q, k)$ of the quark-gluon vertex Eq. (7) contains ghostdressing factors as (partly) implied by its Slavnov-Taylor identity. The combined dressing of the quark-gluon vertex and the full gluon propagator in the quark DSE then provides enough interaction strength to generate dynamical quark masses of the order of $M(0) \approx 350 \mathrm{MeV}$ in agreement with phenomenology. Furthermore a chiral condensate of the order $(-\langle\bar{\Psi} \Psi\rangle)^{-1 / 3} \approx 300$ $\mathrm{MeV}$ has been obtained, which strongly favours the standard counting rules in chiral perturbation theory.

The results for the quenched quark mass function $M\left(p^{2}\right)$ and the wave function $Z_{Q}\left(p^{2}\right)$ are compared to the quenched lattice results of Ref. [16] in Fig. 3. The overall qualitative and quantitative agreement between both approaches is very good. The DSE results are within the bounds given by the two different formulations of fermions on the lattice.

Including the backreaction of the quarkpropagator on the ghost and gluon system leads to a coupled set of three Dyson-Schwinger equations for the propagators of QCD. These equations have been solved in [14] and allowed a prediction of possible effects of unquenching QCD on the propagators. As can be seen from Fig. 3 including $N_{f}=3$ chiral quarks in the gluon DSE hardly changes the results for the quark propagator. The slight enhancement in the perturbative tail of the mass function is in perfect accordance with the expected change of the anomalous dimension. The chiral condensate is nearly unaffected. It will be interesting to compare these results to unquenched lattice calculations when available.

Unquenched lattice results for the gluon propagator including the effects of two light (up-) and one heavy (strange-) quark have been published recently [18] and are compared to the corresponding results from our DSE-approach in Fig. 4. The screening effect from the quark loop is clearly visible in the lattice results for momenta $p$ larger than $p=0.5 \mathrm{GeV}$ : the gluonic self interaction becomes less important in this region and the gluon dressing increases. Although to a somewhat less extent, this effect can also be seen in the DSEapproach. As mentioned above not all effects from the gluonic self interaction are accounted for in the DSE truncation. When this part of the gluon interaction becomes less dominant in the unquenched case, both the lattice and the DSEapproach agree very well on a quantitative level, provided similar bare quark masses are taken into account. In the chiral limit the screening effect of the quark loop becomes even stronger as can be seen from the DSE-results in Fig. 4. This is certainly expected as the energy needed to create a quark pair out of the vacuum becomes smaller with decreasing bare quark mass.

Both, the lattice calculations and the Green's functions approach agree in the fact that unquenching does not affect the extreme infrared of the ghost and gluon propagators. Again, this is easily explained from dynamical chiral symmetry breaking: there is not enough energy to generate a quark pair from the vacuum below a certain threshold, and the quark degrees of freedom decouple from the Yang-Mills sector of the theory. 


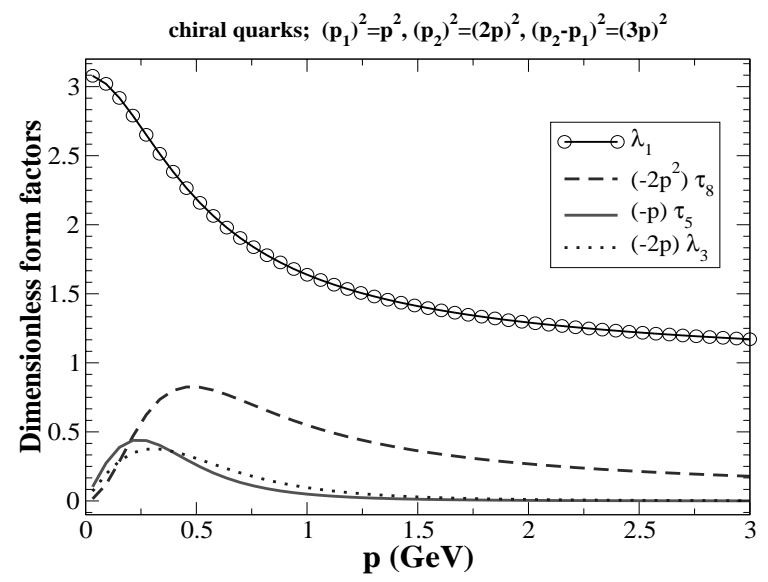

Figure 5. The leading tensor structures of the quark gluon vertex from the vertex DSE with chiral quarks.

\section{THE QUARK-GLUON VERTEX}

As mentioned in the introduction, the quarkgluon vertex plays an important role in both, understanding confinement from the quark-gluon interaction and providing the bridge between coloured quarks and gluons, and their colourless bound states, the hadrons. Therefore it is desirable to determine its properties in a selfconsistent calculation. As a first step in this direction we present a calculation of the components of the quark-gluon vertex from its DSE (also see Ref.[21]). Our calculation is not selfconsistent yet: we use the quark, ghost and gluon propagators presented in the last section as input on the right hand side of the vertex DSE without backcoupling the vertex to the quark equation. Furthermore we only employ the $\gamma_{\mu}$-part of the vertex in all loops of the vertex DSE. Nevertheless we believe that our results are meaningful as the propagators used are close to their respective counterparts on the lattice. Furthermore from the structure of the vertex DSE one finds indications that selfconsistency effects could play a major role in the infrared but are small in the medium and large momentum regime.

With these caveats in mind we present our results in Figs. 5 and 6 . The complete vertex can be decomposed in a basis of twelve tensor structures, $\Gamma_{\mu}=\frac{i}{g}\left(\sum_{i=1}^{4} \lambda_{i} L_{i \mu}+\sum_{i=1}^{8} \tau_{i} T_{i \mu}\right)[22]$. Here

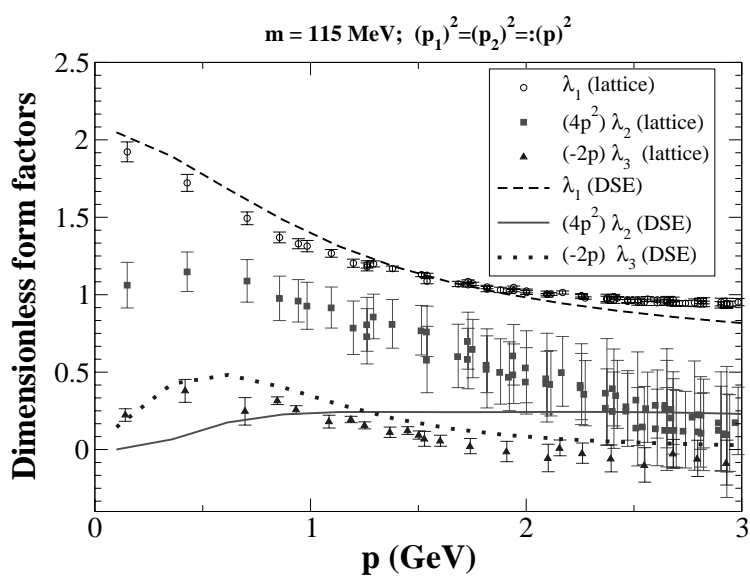

Figure 6. Tensor structures of the quark gluon vertex from the vertex DSE with a massive quark compared to lattice calculations [22].

we show only five of these components, namely the ones multiplying the tensors

$L_{1 \mu}=\gamma_{\mu}$,

$L_{2 \mu}=-\left(\not p_{1}+\not p_{2}\right)\left(p_{1}+p_{2}\right)_{\mu}$,

$L_{3 \mu}=i\left(p_{1}+p_{2}\right)_{\mu}$,

$T_{5 \mu}=i \sigma_{\mu \nu}\left(p_{2}-p_{1}\right)^{\nu}$,

$T_{8 \mu}=-\gamma_{\mu} \sigma_{\lambda \nu} p_{1}^{\lambda} p_{2}^{\nu}-\not p_{1} p_{2 \mu}+\not p_{2} p_{1 \mu}$,

with the quark momenta $p_{1}$ and $p_{2}$. The (dimensionless) four leading structures in the chiral limit are given in Fig. 5. Although the $L_{1}$ - piece is dominating, sizeable admixtures from other tensor structures occur. This is also found in a recent model study [23] and it is expected, as the quark-gluon vertex provides one of the underlying structures from which the rich structure of phenomenological quark potentials (see e.g. [24]) should be ultimately derived.

Our results for a massive quark of $m_{0}=115$ $\mathrm{MeV}$ are compared with the available lattice results in Fig. 6. Even without selfconsistency in the DSEs we obtain very nice agreement for the vector and the scalar tensor components, $L_{1}$ and $L_{3}$. On the other hand, the infrared behaviour of the $L_{2}$-piece is in obvious disagreement. Firm conclusions, however, cannot be drawn before lattice data with smaller errors as well as a selfconsistent DSE solution are available. 


\section{OUTLOOK}

In the last years a consistent picture of the infrared behaviour of Landau gauge QCD has emerged. Evidence from the Green's functions approach and lattice calculations suggests that the Faddeev-Popov determinant (i.e. the ghost degrees of freedom) is dominant in the infrared and provide the long range interaction in QCD. The gluon propagator is finite or even vanishing in the infrared. Taken at face value this behaviour provides a problem for confinement: Simple onegluon exchange with a bare quark-gluon vertex cannot account for the linear rising potential between two static colour sources. Infrared singularities present in the dressed quark-gluon vertex could resolve this issue. Whether this is indeed the case or a more sophisticated mechanism is at work remains an open problem for the future.

\section{ACKNOWLEDGMENTS}

We are grateful to M. Bhagwat, H. Gies, C. Roberts, J. Skullerud, P. Tandy, A. Williams, and D. Zwanziger for helpful discussions.

This work has been supported by the Deutsche Forschungsgemeinschaft (DFG) under contracts Al 279/3-3, Al 279/3-4, Fi 970/2-1 and GRK683. F.L. thanks the German DAAD and Univ. Complutense for support and the members of the Institute for Theoretical Physics of the University of Tübingen for their hospitality during his visit, when the project reported in section 4 has been started.

We thank A. Kizilersü, A. Thomas and A. Williams for the organisation of this exceptional conference QCD DOWN UNDER.

\section{REFERENCES}

1. P. Maris and C. D. Roberts, Int. J. Mod. Phys. E12 (2003) 297.

2. R. Alkofer and L. von Smekal, Phys. Rept. 353 (2001) 281.

3. C. D. Roberts and S. M. Schmidt, Prog. Part. Nucl. Phys. 45 (2000) S1.

4. L. von Smekal, R. Alkofer and A. Hauck, Phys. Rev. Lett. 79 (1997) 3591; Annals Phys. 267 (1998) 1.
5. C. S. Fischer and R. Alkofer, Phys. Lett. B536 (2002) 177; R. Alkofer, C. S. Fischer and L. von Smekal, Acta Phys. Slov. 52 (2002) 191.

6. C. S. Fischer, R. Alkofer and H. Reinhardt, Phys. Rev. D65 (2002) 094008.

7. C. Lerche and L. von Smekal, Phys. Rev. D65 (2002) 125006.

8. D. Zwanziger, Phys. Rev. D65 (2002) 094039

9. P. Watson and R. Alkofer, Phys. Rev. Lett. 86 (2001) 5239 .

10. N. Nakanishi and I. Ojima, "Covariant Operator Formalism Of Gauge Theories And Quantum Gravity," World Sci. Lect. Notes Phys. 27 (1990) 1.

11. T. Kugo and I. Ojima, Prog. Theor. Phys. Suppl. $66 \quad(1979)$ 1.

12. D. Zwanziger, Phys. Rev. D69 (2004) 016002.

13. J. M. Pawlowski, D. F. Litim, S. Nedelko and L. von Smekal, arXiv:hep-th/0312324; C. S. Fischer and H. Gies, in preparation.

14. C. S. Fischer and R. Alkofer, Phys. Rev. D67 (2003) 094020.

15. D. V. Shirkov and I. L. Solovtsov, Phys. Rev. Lett. 79 (1997) 1209.

16. P. O. Bowman, U. M. Heller and A. G. Williams, Phys. Rev. D66 (2002) 014505; J. B. Zhang et al., arXiv: heplat/0301018.

17. F. D. Bonnet et al., Phys. Rev. D64 (2001) 034501.

18. P. O. Bowman et al., arXiv:hep-lat/0402032.

19. D. C. Curtis and M. R. Pennington, Phys. Rev. D42 (1990) 4165.

20. R. Alkofer, W. Detmold, C. S. Fischer and P. Maris, arXiv:hep-ph/0309077; R. Alkofer, W. Detmold, C. S. Fischer and P. Maris, arXiv:hep-ph/0309078.

21. M. S. Bhagwat et al., arXiv:nucl-th/0403012.

22. J. I. Skullerud et al., JHEP 0304 (2003) 047.

23. P. Watson, W. Cassing and P. C. Tandy, arXiv:hep-ph/0406340.

24. D. Ebert, R. N. Faustov and V. O. Galkin, Phys. Rev. D67 (2003) 014027. 